\title{
Containing Coronavirus (COVID-19) Spread in an Oncology Day Care Facility in India
}

Sir,

Coronavirus (COVID-19) first identified in late December 2019 at Hubei province of China and had spread globally in mammoth proportions. ${ }^{[1]}$ This pandemic is one of the greatest challenge medical fields that has ever faced. The disease by itself and measures taken to contain it has resulted in hampering health-care services to nonpandemic diseases. Reports of reduced hospital admissions for acute coronary syndromes and increased out of the hospital cardiac arrests reflect a compromised health system..$^{[2,3]}$ Sustaining non COVID-19 facility in current pandemic has become a big challenge. The risk of transmission of infection among health-care personnels (HCPs) from HCPs to patients and requirement of quarantine of potentially exposed HCPs pose further burden on already constrained system. Continuing oncology services in the current pandemic have become even more difficult considering higher mortality rates of COVID-19 in cancer patients. ${ }^{[4,5]}$ Most guidelines have been revised and suggested tailored strategies in situations where the risk of infection outweighs the benefit of treatment. ${ }^{[6]}$

In the Indian context, with an existing strained health-care system, providing optimum oncology care is challenging. The maintenance of special care facilities such as daycare chemotherapy centers, radiation facilities, and palliative care units require special emphasis. These centers are like a catchment area in view of compromised immunity of patients and high turnover of people at any given point of time. Even a single positive case among them leads to devastating consequences, including interruptions of the services. Daycare chemotherapy facility is an important component of oncology care. In this report, we share our experience of containing a potential spread and measures taken to reopen the facility in short time. This kind of model can be followed in various similar facilities.

\section{The problem}

Our oncology center, DR. B. R. Ambedkar Institute Rotary Cancer Hospital All India Institute of Medical Sciences (AIIMS), is one of the largest cancer centers in North India registering 13,000 new cancer cases every year. Our chemotherapy day care facility caters around 120 patients per day during the pre-COVID-19 times. Currently, it is managing almost 50-60 patients per day due to several travel restrictions and countrywide lockdown. One oncology nurse at day-care chemotherapy facility developed fever and cough for 1 day. She attended patient care services 1 day before the onset of symptoms. After the development of symptoms, she was tested for COVID-19 by RT-PCR (reverse transcription polymerase chain reaction) and found to be positive. She was isolated according to the standard operating procedure (SOP), for mitigating further spread. During going in close contact with patients, HCPs, including the index case, were using personal protective equipment (PPE) which included N95 respirator mask, gown, gloves, and head cap. As per hospital policies during the pandemic, all the patients in day care either worn a cloth or triple-layer surgical mask.

\section{The response}

The mitigation measures were carried out as per SOPs in the hospital. The preparedness of the institute that was already in the place helped in execution of the plan smoothly as below. These measures are depicted in Table 1 which includes contact tracing, testing, quarantine, treatment, and sanitation of the facility.

Table 1: Strategies implemented at containment of spread

\begin{tabular}{ll}
\hline Measures & Components \\
\hline Preparedness & Droplet infection control practices in place \\
& Trained HCP \\
& PPE and N95 for HCP \\
& Mask for all patients \\
& Spacing of daycare beds \\
& Display of hand hygiene and cough etiquette \\
& measures \\
& Alcohol hand wash in place \\
& Backup plan with strategy of 2 treatment teams \\
& with one team as standby \\
& Screening of patients for symptoms of \\
& COVID-19 before reaching daycare facility \\
& Ramped up testing capabilities \\
& Separate quarantine and isolation facility \\
& Special tracing teams \\
Isolation of positive case \\
Qontact tracing \\
and isolation & Testing of first contacts between day-5 and \\
desting & Tele-consulted daily for onset of symptoms \\
Telemedicine & Growth factor supplementation \\
& Delay of further chemotherapy (in case of \\
Sanitation & multiday schedule) \\
& Disinfection of entire facility twice with \\
& Ramigation [Appendix 1] \\
& Random swabs taken and tested \\
\hline
\end{tabular}

$\overline{\mathrm{HCP}}$ - Health care personnel; PPE - Personal protective equipment; COVID - Corona virus 


\section{Preparedness in place}

In view of ensuing pandemic, such a scenario was anticipated, and few measures were taken. All the HCP received training in infection control practices, donning, and doffing of PPE. As an institutional practice, all HCPs wore a N95 respirator and patients with a surgical/cloth mask. Information regarding the hand wash techniques, cough etiquette, etc., was displayed throughout the hospital. Alcohol hand wash facility was widely provided. All the patients reaching day-care facility were screened for COVID-19 symptoms. To ensure adequate spacing at the facility, alternate bed was kept vacant. To prevent a situation wherein, quarantine of entire treatment unit resulting in closing institute, we have two teams working alternatively for 7 days each. In case any unit gets quarantined, another team takes over so that patient care is not compromised. Furthermore, separate areas are demarcated for managing COVID-19 patients, thus preventing the admixture. Specialized teams are deputed for contact tracing and quarantine facilitates with round the clock helpline number availability. Testing capacity of the center for COVID-19 has been ramped up, which forms the core of mitigation measures. Telemedicine facility has been initiated and operationalized for facilitating the patient care and effective monitoring of quarantined contacts.

\section{Tracing, testing and treatment}

Oncology nurse was shifted to an isolation facility and was given symptomatic management. Spouse of the index patient who was also a HCP and two children were quarantined and tested. Contact tracing was initiated, and a list of individuals who had contact with her was prepared. This included a total of 43 among them two were oncology trainees, 18 nursing staff, 20 patients, and 3 family members. The HCP were put off roster and advised home quarantine for 14 days. These are depicted in Table 2. They were actively followed up for the development of symptoms. The patients were contacted through telemedicine facility. All the contacts were advised RT-PCR testing between day 5 and day 14 of contact. Out of 43 contacts, we could do testing in 34 of them. The remaining nine individuals could not be tested because they could not reach in the hospital due to lockdown measures. They were advised strict quarantine at home. Out of the 34 contacts tested, 3 were positive. Two children of the index nurse were positive, and another day care oncology nurse was positive. Two children had mild symptoms, whereas the nurse was completely asymptomatic. They were shifted to isolation facility at our institute. All of them recovered and discharged once they have been tested negative on two consecutive occasions $24 \mathrm{~h}$ apart.

\section{Lacuna identified}

During the process of contact, tracing exercise potential risk was identified for transmission. At the time of break hours, HCPs were noticed to be in groups of 4-5 having meals together which might have resulted in unprotected exposure. Subsequently, a staggered break time and duty time policy were implemented to minimize such potential risks.

\section{Reopening of facility}

After the detection of positive case, the facility was closed, and the measures as described above were done. The entire facility was sanitized with 1\% sodium hypochlorite solution twice and later followed by fumigation. Random swabs taken were negative. The facility was reopened after $48 \mathrm{~h}$ with the second team which was on stand-by.

\section{Current status}

As of the date of submission for the publication, the two COVID-19 cases were discharged and all the contacts completed their quarantine period of 14 days. No new cases were identified. All the quarantined staff resumed their duties.

\section{Conclusion}

Our case emphasizes the requirement of preparedness for effective management in the current pandemic. With preplanned coordinated measures and SOPs in place, we could not only contain the spread, but also reopen the facility within 2 days so that patient care was not hampered. Testing of contacts, even asymptomatic is critical. Identification of all asymptomatic carriers is the cornerstone in controlling COVID-19. Universal face-cover and social distancing should be the norm. Adapting to a new normal and learning to live with COVID-19 by changing socialization habits such as group activities are required. Health-care working models with two teams working alternatively will help to sustain hospitals in these testing times.

\begin{tabular}{llccl}
\hline & & Table 2: Contact tracing & \\
\hline Category & Description $(\boldsymbol{n})$ & Testing & Positive for COVID-19 & Measures taken \\
\hline Family & Spouse: 1 & $3 / 3$ & 2 & Isolation until 2 consecutive tests negative \\
& Children: 2 & & 1 & Quarantine for 14 days \\
HCP & Oncology trainees: 2 & $20 / 20$ & 0 & Quarantine for 14 days \\
& Oncology nurse: 18 & & & \\
Patients & Hematological malignancies: 8 & $11 / 20$ & & \\
& Solid malignancy: 12 & &
\end{tabular}

HCP - Health care personnel; COVID - Corona virus 


\section{Financial support and sponsorship}

Nil.

\section{Conflicts of interest}

There are no conflicts of interest.

\section{Santosh Kumar Chellapuram, Prabhat S Malik, Lalit Kumar}

Department of Medical Oncology, All India Institute of Medical Sciences, Dr B.R.A. Institute-Rotary Cancer Hospital, New Delhi, India

Address for correspondence: Prof. Lalit Kumar, Department of Medical Oncology, All India Institute of Medical Sciences, Dr B.R.A. Institute-Rotary Cancer Hospital, New Delhi, India. E-mail:lalitaiims@yahoo.com

Submitted: 01-Jun-2020

Revised: 26-Jun-2020

Accepted: 06-Jul-2020

Published: 29-Aug-2020

\section{References}

1. Zhu N, Zhang D, Wang W, Li X, Yang B, Song J, et al. A novel coronavirus from patients with pneumonia in China, 2019. N Engl J Med 2020;382:727-33.

2. Baldi E, Sechi GM, Mare C, Canevari F, Brancaglione A, Primi R, et al. Out-of-hospital cardiac arrest during the Covid-19 outbreak in Italy [published online ahead of print, 2020 Apr 29]. N Engl J Med 2020;NEJMc2010418.

3. De Filippo O, D'Ascenzo F, Angelini F, Bocchino PP, Conrotto F, Saglietto A, et al. Reduced rate of hospital admissions for ACS during Covid-19 outbreak in Northern Italy. $\mathrm{N}$ Engl J Med 2020;383:88-9.
4. Dai M, Liu D, Liu M, Zhou F, Li G, Chen Z, et al. Patients with cancer appear more vulnerable to SARS-COV-2: A multi-center study during the COVID-19 outbreak. Cancer Discov 2020;CD20-0422. Available from: https://cancerdiscovery.aacrjourn als.org/content/early/2020/04/29/2159-8290.CD-20-0422. [Last accessed on 2020 May 9].

5. Liang W, Guan W, Chen R, Wang W, Li J, Xu K, et al. Cancer patients in SARS-CoV-2 infection: A nationwide analysis in China. Lancet Oncol 2020;21:335-7.

6. Ueda M, Martins R, Hendrie PC, McDonnell T, Crews JR, Wong TL, et al. Managing cancer care during the COVID-19 pandemic: Agility and collaboration toward a common goal. [published online ahead of print, 2020 Mar 20]. J Natl Compr Canc Netw 2020;1-4.

This is an open access journal, and articles are distributed under the terms of the Creative Commons Attribution-NonCommercial-ShareAlike 4.0 License, which allows others to remix, tweak, and build upon the work non-commercially, as long as appropriate credit is given and the new creations are licensed under the identical terms.

\begin{tabular}{|l|l|}
\hline \multicolumn{2}{|c|}{ Access this article online } \\
\hline Quick Response Code: & Website: \\
& www.ijmpo.org \\
\cline { 2 - 2 } & DOI: \\
\hline
\end{tabular}

How to cite this article: Chellapuram SK, Malik PS, Kumar L. Containing coronavirus (COVID-19) spread in an oncology day care facility in India. Indian J Med Paediatr Oncol 2020;41:468-70. 


\section{Appendix}

\section{Appendix: 1}

Decontamination protocol: Detergent + disinfectant + plus fogging with $\mathrm{H}_{2} \mathrm{O}_{2}$

\section{Cleaning agents and disinfectants:}

1. $1 \%$ Sodium Hypochlorite can be used as a disinfectant for cleaning and disinfection

2. The solution should be prepared fresh

3. Leaving the solution for a contact time of at least $10 \mathrm{~min}$ is recommended

4. Alcohol (e. g. isopropyl 70\% or ethyl alcohol 70\%) can be used to wipe down surfaces where the use of bleach is not suitable, e.g., metals.

\section{PPE to wear while carrying out cleaning and disinfection works:}

1. Wear heavy duty/disposable gloves, disposable long-sleeved gowns, eye goggles or a face shield, and a medical mask (please see the PPE document for details)

2. Avoid touching the nose and mouth (goggles may help as they will prevent hands from touching eyes)

3. Disposable gloves should be removed and discarded if they become soiled or damaged, and a new pair worn

4. All other disposable PPE should be removed and discarded after cleaning activities are completed. Eye goggles, if used, should be disinfected after each use, according to the manufacturer's instructions

5. Hands should be washed with soap and water/alcohol-based hand rub immediately after each piece of PPE is removed, following completion of cleaning.

\section{Cleaning guidelines:}

1. Where possible, seal off areas where the confirmed case has visited, before carrying out cleaning and disinfection of the contaminated environmental surfaces. This is to prevent unsuspecting persons from being exposed to those surfaces

2. When cleaning areas where a confirmed case has been, cleaning staff should be attired in suitable PPE. Disposable gloves should be removed and discarded if they become soiled or damaged, and a new pair worn. All other disposable PPE should be removed and discarded, after cleaning activities are completed. Goggles, if used, should be disinfected after each use, according to manufacturer's instructions. Hands should be washed with soap and water immediately after the PPE is removed

3. Mop floor with routinely available disinfectant

4. Wipe all frequently touched areas (e.g. lift buttons, hand rails, doorknobs, arm rests, tables, air/light controls, keyboards, switches, etc.) and toilet surfaces with chemical disinfectants and allow to air dry. 1\% sodium hypochlorite solution can be used. Alcohol can be used for surfaces, where the use of bleach is not suitable

5. Clean toilets, including the toilet bowl and accessible surfaces in the toilet with disinfectant or $1 \%$ sodium hypochlorite solution

6. Wipe down all accessible surfaces of walls as well as blinds with disinfectant or bleach solution

7. Remove curtains/fabrics/quilts for washing, preferably using the hot water cycle. For hot-water laundry cycles, wash with detergent or disinfectant in water at $70^{\circ} \mathrm{C}$ for at least $25 \mathrm{~min}$

8. Discard cleaning items made of cloth and absorbent materials, e. g. mop head and wiping cloths, into biohazard bags after cleaning and disinfecting each area. Wear a new pair of gloves and fasten the double-bagged biohazard bag with a cable tie

9. Disinfect buckets by soaking in disinfectant or bleach solution, or rinse in hot water before filling

10. Disinfectant or $1 \%$ sodium hypochlorite solution should be applied to surfaces using a damp cloth. They should not be applied to surfaces using a spray pack, as coverage is uncertain and spraying may promote the production of aerosols. The creation of aerosols caused by splashing liquid during cleaning should be avoided. A steady sweeping motion should be used when cleaning either floors or horizontal surfaces, to prevent the creation of aerosols or splashing. Cleaning methods that might aerosolize infectious material, such as the use of compressed air, must not be used

11. Biohazard bags should be properly disposed-off, upon completion of the disinfection work.

\section{Frequency of cleaning of surfaces:}

1. High touch surfaces: Disinfection of high touch surfaces like (doorknobs, telephone, call bells, bedrails, stair rails, light switches, wall areas around the toilet) should be done every 3-4 h

2. Low-touch surfaces: For Low-touch surfaces (walls, mirrors, etc.) mopping should be done at least once daily. 


\section{Precautions to take after completing the clean-up and disinfection}

1. Staff should wash their hands with soap and water immediately after removing the PPE, and when cleaning and disinfection work is completed

2. Discard all used PPE in a double-bagged biohazard bag, which should then be securely sealed and labelled

3. The staff should be aware of the symptoms and should report to their occupational health service if they develop symptoms. 